\title{
Parasupersymmetric Quantum Mechanics and Indices of Fredholm Operators
}

\author{
Ali Mostafazadeh* \\ Theoretical Physics Institute, University of Alberta, \\ Edmonton, Alberta, Canada T6G 2J1. \\ June 1996
}

\begin{abstract}
The general features of the degeneracy structure of $(p=2)$ parasupersymmetric quantum mechanics are employed to yield a classification scheme for the form of the parasupersymmetric Hamiltonians. The method is applied to parasupersymmetric systems whose Hamiltonian is the square root of a forth order polynomial in the generators of the parasupersymmetry. These systems are interesting to study for they lead to the introduction of a set of topological invariants very similar to the Witten indices of ordinary supersymmetric quantum mechanics. The topological invariants associated with parasupersymmetry are shown to be related to a pair of Fredholm operators satisfying two compatibility conditions. An explicit algebraic expression for the topological invariants of a class of parasupersymmetric systems is provided.
\end{abstract}

*E-mail: alimos@phys.ualberta.ca 


\section{Introduction}

Perhaps one of the most intriguing aspects of supersymmetry is its relation with the AtiyahSinger index theorem [1]. It was Witten [4] who first recognized this relation in the context of supersymmetric quantum mechanics (SQM). The subsequent developments in this direction have led to supersymmetric proofs of the index theorem [5].

Few years after the publication of the first supersymmetric proofs of the index theorem Rubakov and Spiridonov (R-S) [7] introduced their $(p=2)$-parasupersymmetric quantum mechanics (PSQM). This involved a generalization of the superalgebra of SQM円:

$$
\mathcal{Q}^{2}=[\mathcal{Q}, H]=0, \quad\left\{\mathcal{Q}, \mathcal{Q}^{\dagger}\right\}=2 \kappa H, \quad\left(\kappa \in \mathbb{R}^{+}\right)
$$

namely the parasuperalgebra:

$$
\begin{gathered}
\mathcal{Q}^{3}=0, \quad[\mathcal{Q}, H]=0 \\
\left\{\mathcal{Q}^{2}, \mathcal{Q}^{\dagger}\right\}+\mathcal{Q} \mathcal{Q}^{\dagger} \mathcal{Q}=4 \mathcal{Q} H
\end{gathered}
$$

The latter relations (2) and (3) have since been generalized to arbitrary order $p>2$, by Khare [8], and modified by Beckers and Debergh (B-D) [9]. B-D $(p=2)$-parasuperalgebra is given by Eqs. (2) and

$$
\left[\mathcal{Q},\left[\mathcal{Q}^{\dagger}, \mathcal{Q}\right]\right]=2 \mathcal{Q} H
$$

It is not difficult to check that indeed superalgebra of SQM (11), with $\kappa=2$ and $\kappa=1 / 2$, is a special case of the parasuperalgebras of R-S and B-D, respectively. Given the relation between SQM and topological invariants such as indices of Fredholm operators, the latter observation renders the following questions:

1) Is PSQM related to topological invariants? If so,

2) Are these invariants more general than the indices of Fredholm operators?

In a preceding article [10], it is shown that a careful analysis of the defining parasuperalgebra (for both R-S and B-D types) provides important information on the degeneracy structure of the spectrum of the corresponding systems. In particular, postulating the existence of a parasupersymmetry involution (chirality) operator and supplementing (either of)

\footnotetext{
${ }^{1}$ Here $\kappa$ is a conventional positive constant.
} 
the parasuperalgebra(s) with an additional relation expressing the Hamiltonian in terms of the parasupercharges, namelyt

$$
H=\frac{1}{2}\left[\left(\mathcal{Q} \mathcal{Q}^{\dagger}\right)^{2}+\left(\mathcal{Q}^{\dagger} \mathcal{Q}\right)^{2}-\frac{1}{2}\left(\mathcal{Q} \mathcal{Q}^{\dagger 2} \mathcal{Q}+\mathcal{Q}^{\dagger} \mathcal{Q}^{2} \mathcal{Q}^{\dagger}\right)\right]^{\frac{1}{2}}
$$

one can show that the integer

$$
\begin{aligned}
\Delta^{(p=2)} & :=n^{\pi B}-2 n^{\pi F}=n_{0}^{\pi B}-2 n_{0}^{\pi F}, \quad \text { with } \\
n^{\pi B} & :=\text { number of parabosonic states } \\
n^{\pi F} & :=\text { number of parafermionic states } \\
n_{0}^{\pi B} & :=\text { number of zero energy parabosonic states } \\
n_{0}^{\pi F} & :=\text { number of zero energy parafermionic states }
\end{aligned}
$$

is a topological invariant. Furthermore, it is shown in 10 that $\Delta^{(p=2)}$ is a measure of parasupersymmetry breaking, i.e., the condition $\Delta^{(p=2)} \neq 0$ implies the exactness of parasupersymmetry. In this respect, it is quite similar to the Witten index of supersymmetry. However, unlike the Witten index a mathematical interpretation of this invariant has been lacking until now.

A major difference between SQM and PSQM is that unlike the defining (super)algebra (1) of SQM, the defining (parasuper)algebras (2), (3) and (2), (4) do not provide an expression for the Hamiltonian. In fact, one can easily see that the form of the Hamiltonian in terms of the generators in both R-S and B-D PSQM is not unique. Therefore, a relevant problem is to seek a classification scheme for all possible forms of the PSQM Hamiltonian.

The purpose of the present article is twofold. First it is shown that the developments reported in Ref. [10] can be used, with some additional effort, to devise a classification scheme for the PSQM Hamiltonians. This scheme is then used to study the topological content of $(p=2)$ PSQM in a broader framework and to provide a precise mathematical interpretation for the corresponding topological invariants.

In Sec. 2, a brief discussion of SQM is presented to demonstrate the motivation for the proceeding analysis of PSQM. Sec. 3 summarizes the necessary back ground information on the degeneracy structure of the $(p=2)$ PSQM. This information is then used to address

\footnotetext{
${ }^{2}$ The Hamiltonian (5) was originally proposed by Khare et al. [11] for different purposes.
} 
the classification problem. In particular, the moduli space of all $(p=2)$ PSQM systems admitting a Hamiltonian whose square is a forth order polynomial in the generators of parasupersymmetry, is constructed. Here the subspace of systems for which $\Delta^{(p=2)}$ is a topological invariant is identified. In Sec. 4, these invariants are shown to be related to the indices of a pair of Fredholm operators, thus providing the mathematical interpretation of interest. Sec. 5 includes the concluding remarks.

\section{SQM and the Index Theorem}

The main ingredient of SQM which makes its relation with the index theory possible, is its simple degeneracy structure. More precisely, the degeneracy structure of the spectrum of any supersymmetric quantum mechanical system is determined using only the defining superalgebra (1) and the properties of the supersymmetry involution (chirality) operator $\tau$ :

$$
\tau^{2}=1, \tau^{\dagger}=\tau,\{\mathcal{Q}, \tau\}=0
$$

In Eqs. (1) and (7), $\mathcal{Q}$ stands for (one of) the generator(s) of supersymmetry, $\mathcal{Q}^{\dagger}$ is its adjoint, and $H$ is the Hamiltonian. The chirality operator $\tau$ induces a double grading of the Hilbert space, $\mathcal{H}=\mathcal{H}_{+} \oplus \mathcal{H}_{-}$, where

$$
\mathcal{H}_{ \pm}:=\{\psi \in \mathcal{H}: \tau \psi= \pm \psi\}
$$

The superalgebra (1) can be employed to show that the energy spectrum is non-negative and that each positive energy state of definite chirality is accompanied with another state of the same energy and opposite chirality, [4, 10]. In this sense, one says that the positive energy levels are doubly degenerate.

Introducing the self-adjoint generators:

$$
Q_{1}=\frac{1}{\sqrt{2}}\left(\mathcal{Q}+\mathcal{Q}^{\dagger}\right), \quad Q_{2}=\frac{-i}{\sqrt{2}}\left(\mathcal{Q}-\mathcal{Q}^{\dagger}\right)
$$

one rewrites the superalgebra (11) in the form:

$$
\begin{aligned}
\left\{Q_{1}, Q_{2}\right\} & =0 \\
Q_{1}^{2}=Q_{2}^{2} & =H
\end{aligned}
$$




$$
\begin{aligned}
& {\left[Q_{1}, H\right]=0,} \\
& {\left[Q_{2}, H\right]=0,} \\
& \left\{Q_{1}, \tau\right\}=0, \\
& \left\{Q_{2}, \tau\right\}=0, \\
& \tau^{2}=1 \quad, \quad \tau^{\dagger}=\tau .
\end{aligned}
$$

In view of (12), one can use the eigenvalues $E$ and $q_{1}= \pm \sqrt{E}$ of $H$ and $Q_{1}$, to label the states. Here we choose not to include any other quantum numbers. Their presence will not interfere with the arguments presented in this article.

For each positive energy level $(E>0)$, the $\{|E, \pm \sqrt{E}\rangle\}$ basis may be used to yield matrix representations of the relevant operators [10]. Alternatively, one may adapt a basis in which $H$ and $\tau$ are diagonal. In such a basis one has:

$$
\begin{aligned}
\left.Q_{1}\right|_{\mathcal{H}_{E}} & =\sqrt{\kappa E}\left(\begin{array}{cc}
0 & -i \\
i & 0
\end{array}\right)=\sqrt{\kappa E} \sigma_{2}, \\
\left.Q_{2}\right|_{\mathcal{H}_{E}} & =\sqrt{\kappa E}\left(\begin{array}{ll}
0 & 1 \\
1 & 0
\end{array}\right)=\sqrt{\kappa E} \sigma_{1}, \\
\left.\tau\right|_{\mathcal{H}_{E}}=\left(\begin{array}{cc}
1 & 0 \\
0 & -1
\end{array}\right) & =\sigma_{3},\left.\quad H\right|_{\mathcal{H}_{E}}=E\left(\begin{array}{ll}
1 & 0 \\
0 & 1
\end{array}\right) .
\end{aligned}
$$

where $\mathcal{H}_{E}$ denotes the eigenspace associated with the eigenvalue $E$ and $\sigma_{i}, i=1,2,3$, are Pauli matrices. The fact that $\operatorname{trace}\left(\left.\tau\right|_{\mathcal{H}_{E}}\right)=0$ is the very reason for the topological invariance of the Witten index 四:

$$
\begin{aligned}
\operatorname{index}_{W} & :=\operatorname{trace}(\tau)=n^{B}-n^{F}=n_{0}^{B}-n_{0}^{F} \\
n^{B} & :=\text { number of bosonic states } \\
n^{F} & :=\text { number of fermionic states } \\
n_{0}^{B} & :=\text { number of zero energy bosonic states } \\
n_{0}^{F} & :=\text { number of zero energy fermionic states }
\end{aligned}
$$

Eq. (17) serves as a motivation for relating the Witten index with the analytic indices of Fredholm operators. To demonstrate this relationship, first one introduces the representation

$$
\mathcal{H}=\left(\begin{array}{l}
\mathcal{H}_{+} \\
\mathcal{H}_{-}
\end{array}\right)
$$


of the Hilbert space in which $\tau$ is (block-)diagonal. To obtain the representations of $Q_{i}$ $(i=1,2)$, one appeals to Eqs. (14) and (15). These together with (17) suggest:

$$
Q_{1}=\left(\begin{array}{cc}
0 & -i D_{1}^{\dagger} \\
i D_{1} & 0
\end{array}\right), Q_{2}=\left(\begin{array}{cc}
0 & D_{2}^{\dagger} \\
D_{2} & 0
\end{array}\right)
$$

where $D_{i}: \mathcal{H}_{+} \rightarrow \mathcal{H}_{-}, i=1,2$ are a couple of operators acting on $\mathcal{H}_{+}$and $D_{i}^{\dagger}$ are their adjoints. Enforcing the superalgebra, namely Eqs. (10) and (11), this representation leads to the following set of compatibility conditions for $D_{i}$ :

$$
\begin{aligned}
& D_{1}^{\dagger} D_{2}=D_{2}^{\dagger} D_{1}, \\
& D_{1} D_{2}^{\dagger}=D_{2} D_{1}^{\dagger}, \\
& D_{1}^{\dagger} D_{1}=D_{2}^{\dagger} D_{2}, \\
& D_{1} D_{1}^{\dagger}=D_{2} D_{2}^{\dagger} .
\end{aligned}
$$

In view of Eqs. (11), (23), and (24), the Hamiltonian takes the form:

$$
H=\left(\begin{array}{cc}
D_{i}^{\dagger} D_{i} & 0 \\
0 & D_{i} D_{i}^{\dagger}
\end{array}\right)
$$

The latter relation together with Eq. (18) and the identities:

$$
\operatorname{ker}\left(D_{i}^{\dagger} D_{i}\right)=\operatorname{ker}\left(D_{i}\right), \quad k e r\left(D_{i} D_{i}^{\dagger}\right)=\operatorname{ker}\left(D_{i}^{\dagger}\right)
$$

lead to the desired result [4], namely

$$
\operatorname{index}_{W}=\operatorname{dim}\left(\operatorname{ker} D_{i}\right)-\operatorname{dim}\left(\operatorname{ker} D_{i}^{\dagger}\right)
$$

for either of $i=1,2$. In fact, Witten chooses $D_{1}=D_{2}$ to satisfy the compatibility conditions (21) - (24). If If now one identifies $\mathcal{H}_{ \pm}$with abstract inner product (Hilbert) spaces $\Gamma_{1}$ and $\Gamma_{2}$, and $D_{i}: \Gamma_{1} \rightarrow \Gamma_{2}$ with two (parameter dependent) Fredholm operators, then Eq. (27) implies:

$$
\operatorname{index}_{W}=\operatorname{index}^{\text {Analytic }}\left(D_{i}\right)
$$

\footnotetext{
${ }^{3}$ Note that this is not a necessary condition for satisfying (21) - (24).
} 
for both $i=1,2$. In particular, one can choose $\Gamma_{a}(a=1,2)$ to be spaces of smooth sections of a pair of complex Hermitian vector bundles $E_{a}$ and $D_{i}$ a pair of elliptic differential operators. Then, one has:

$$
\operatorname{index}_{W}=\operatorname{index}^{\text {Atiyah-Singer }}\left(D_{i}\right) \text {, }
$$

where by the Atiyah-Singer index, we mean the topological index introduced by Atiyah and Singer [3]. Eq. (29) is proven for twisted Dirac operators and other classical elliptic operators using the path integral techniques. The former result together with a result of K-theory lead to a proof of the general index theorem, [5, 6].

\section{R-S PSQM and the Classification Problem}

Ref. [10] presents a detailed analysis of both the R-S and the B-D $(p=2)$ PSQM. Here the relevant results are quoted without proof for brevity. As demonstrated in [10 the analysis of the B-D PSQM is quite analogous to the R-S PSQM. This analogy also extends to the subject of this article. Hence the results concerning the B-D PSQM will not be explicitly mentioned.

Consider the R-S parasuperalgebra (2), (3) written in terms of the self-adjoint generators (9):

$$
\begin{array}{r}
Q_{1}^{3}-\left\{Q_{1}, Q_{2}^{2}\right\}-Q_{2} Q_{1} Q_{2}=0 \\
Q_{2}^{3}-\left\{Q_{2}, Q_{1}^{2}\right\}-Q_{1} Q_{2} Q_{1}=0 \\
{\left[Q_{1}, H\right]=\left[Q_{2}, H\right]=0} \\
Q_{1}^{3}=2 Q_{1} H \\
Q_{2}^{3}=2 Q_{2} H
\end{array}
$$

These relations are sufficient to prove the following statements [10]:

1) In general, the spectrum consists of both negative and non-negative energy eigenvalues.

2) The negative and zero energy eigenvalues are non-degeneratef

\footnotetext{
${ }^{4}$ Here degeneracy refers to the eigenvalues of one of the self-adjoint charges, say $Q_{1}$.
} 
3) The positive energy levels may be non-degenerate, doubly degenerate or triply degenerate. The doubly degenerate levels consist of a pair of odd and even chirality states, where as the triply degenerate levels involve two even (resp. odd) and one odd (resp. even) states.

4) Consider an arbitrary degenerate energy level $E$ and denote the corresponding degeneracy subspace by $\mathcal{H}_{E}$. Then in a basis where $Q_{1}$ is diagonal, one has the following matrix representations for the relevant operators:

a) For doubly degenerate levels:

$$
\begin{gathered}
\left.Q_{1}\right|_{\mathcal{H}_{E}}=\sqrt{2 E} \sigma_{3},\left.\quad Q_{2}\right|_{\mathcal{H}_{E}}=\sqrt{2 E} \sigma_{1}, \\
\left.\tau\right|_{\mathcal{H}_{E}}=\eta \sigma_{1}
\end{gathered}
$$

where $\sigma_{i}$ are Pauli matrices and $\eta= \pm$.

b) For triply degenerate levels:

$$
\begin{gathered}
\left.Q_{1}\right|_{\mathcal{H}_{E}}=\sqrt{2 E}\left(\begin{array}{ccc}
1 & 0 & 0 \\
0 & 0 & 0 \\
0 & 0 & -1
\end{array}\right)=\sqrt{2 E} J_{3}^{(1)}, \\
\left.Q_{2}\right|_{\mathcal{H}_{E}}=\sqrt{2 E}\left(\begin{array}{ccc}
0 & \frac{\zeta}{\sqrt{2}} & -i \epsilon \sqrt{1-\zeta^{2}} \\
\frac{\zeta}{\sqrt{2}} & 0 & \frac{\zeta}{\sqrt{2}} \\
i \epsilon \sqrt{1-\zeta^{2}} & \frac{\zeta}{\sqrt{2}} & 0
\end{array}\right) \\
=\sqrt{E} \zeta J_{1}{ }^{(1)}+i \epsilon \sqrt{2 E\left(1-\zeta^{2}\right)}\left(\begin{array}{ccc}
0 & 0 & -1 \\
0 & 0 & 0 \\
1 & 0 & 0
\end{array}\right) \\
\left.\tau\right|_{\mathcal{H}_{E}}=\left(\begin{array}{ccc}
0 & 0 & \tilde{\eta} \\
0 & \eta & 0 \\
\tilde{\eta} & 0 & 0
\end{array}\right),
\end{gathered}
$$

where $\zeta \in[0,1], \epsilon, \eta, \tilde{\eta}= \pm$ are numerical parameters with $\zeta \neq 0 \Rightarrow \tilde{\eta}=-\eta$, and $J_{i}{ }^{(1)}$, with $i=1,2,3$, are the three dimensional $(j=1)$ representation of the generators of $S U(2)$. 
5) The non-degenerate energy eigenstates correspond to the zero eigenvalue of $Q_{1}$. Indeed they are annihilated by both $Q_{1}$ and $Q_{2}$.

In Ref. [10], it is argued that in order to define an analog of the Witten index of SQM, one must focus on PSQM systems which involve only non-negative energy levels and triply degenerate positive energy levels. It is also shown in [10] that postulating a particular form for the Hamiltonian, namely Eq. (5), originally suggested by Khare, et al [11] for different purposes, one realizes the necessary conditions to define the topological invariant $\Delta^{(p=2)}$ of Eq. (6). In this case, the parameter $\zeta$ of Eqs. (36) is forced to take the value 1.

An important observation regarding this matrix representations is that any Hamiltonian $H=H\left(Q_{1}, Q_{2}\right)=H\left(\mathcal{Q}, \mathcal{Q}^{\dagger}\right)$ which satisfies (2) and (3) must necessarily respect the above matrix representations. Therefore, these representations can be used to identify admissible forms of the Hamiltonian. In other words, in order to classify all the R-S PSQM Hamiltonians, one must first consider the most general expression for $H$ and enforce the matrix representations dictated by R-S PSQM. The only guideline for determining this general form is the fact that the dimension of the generators $\mathcal{Q}$ and $\mathcal{Q}^{\dagger}$ is the square root of that of the Hamiltonian (energy). Therefore, in general one must consider the following form:

$$
H_{N}=\sum_{j=1}^{N}\left[P_{j}\left(\mathcal{Q}, \mathcal{Q}^{\dagger}\right)\right]^{1 / j},
$$

where $N$ is an arbitrary positive integer and $P_{j}\left(\mathcal{Q}, \mathcal{Q}^{\dagger}\right)$ is a polynomial in $\mathcal{Q}$ and $\mathcal{Q}^{\dagger}$ of order $2 j$ with the provision that $P_{j}^{\dagger}=P_{j}$. The desired classification scheme is therefore inductive in nature. For each $N \geq 1$ one needs to write down the most general self-adjoint polynomials $P_{j}$ with $j \leq N$ and then enforce the matrix representations. This in turn leads to a series of matrix equations among the coefficients of these polynomials. The solutions of these equations determine the moduli space of the corresponding class of the R-S PSQM Hamiltonians $H_{N}$.

In particular the classification of the Hamiltonians whose $j$-th power is a polynomial $P_{j}\left(\mathcal{Q}, \mathcal{Q}^{\dagger}\right)$ of order $2 j$ is equivalent to solving a set of equations which are algebraic in the coefficients of $P_{j}$ and rational in $\zeta$ and $\epsilon \sqrt{1-\zeta^{2}}$. Eq. (5) is a particular example of such a Hamiltonian.

In the remainder of this section, first the system of Eq. (5) is generalized to systems whose Hamiltonian is square root of a forth order polynomial in the generators, $H=\sqrt{P_{2}\left(\mathcal{Q}, \mathcal{Q}^{\dagger}\right)}$. 
This leads to a class of PSQM systems whose spectra consist only of the non-degenerate zero energy and degenerate positive energy levels. Next a classification of all such systems is carried out and the subclass which lacks the doubly degenerate positive energy levels is identified. The latter consists of the systems for which $\Delta^{(p=2)}$ of Eq. (6) is a topological invariant.

Consider, the most general self-adjoint Hamiltonian $H$ whose square is a forth order polynomial in the generators $\mathcal{Q}$ and $\mathcal{Q}^{\dagger}$ of parasupersymmetry. Since according to (2), $\mathcal{Q}^{3}=0$, one has the following most general form:

$$
\begin{aligned}
H= & {\left[C_{1} \mathcal{Q}^{2} \mathcal{Q}^{\dagger 2}+C_{2} \mathcal{Q}^{\dagger 2} \mathcal{Q}^{2}+C_{3}\left(\mathcal{Q} \mathcal{Q}^{\dagger}\right)^{2}+C_{4}\left(\mathcal{Q}^{\dagger} \mathcal{Q}\right)^{2}+\right.} \\
& C_{5}\left(\mathcal{Q} \mathcal{Q}^{\dagger 2} \mathcal{Q}+\mathcal{Q}^{\dagger} \mathcal{Q}^{2} \mathcal{Q}^{\dagger}\right)+C_{6}\left(\mathcal{Q}^{2} \mathcal{Q}^{\dagger} \mathcal{Q}+\mathcal{Q}^{\dagger} \mathcal{Q} \mathcal{Q}^{\dagger 2}\right)+ \\
& \left.C_{7}\left(\mathcal{Q} \mathcal{Q}^{\dagger} \mathcal{Q}^{2}+\mathcal{Q}^{\dagger 2} \mathcal{Q} \mathcal{Q}^{\dagger}\right)\right]^{1 / 2}
\end{aligned}
$$

where $C_{k}, k=1, \cdots 7$ are real coefficients. In view of the defining parasuperalgebra (3), this relation may be simplified to yield:

$$
\begin{aligned}
H^{2}= & C_{1} \mathcal{Q}^{2} \mathcal{Q}^{\dagger 2}+\left(C_{2}-2 C_{5}\right) \mathcal{Q}^{\dagger 2} \mathcal{Q}^{2}+C_{3}\left(\mathcal{Q} \mathcal{Q}^{\dagger}\right)^{2}+ \\
& \left(C_{4}-2 C_{5}\right)\left(\mathcal{Q}^{\dagger} \mathcal{Q}\right)^{2}+\left[8 C_{5} \mathcal{Q}^{\dagger} \mathcal{Q}+4 C_{7}\left(\mathcal{Q}^{2}+\mathcal{Q}^{\dagger 2}\right)\right] H+ \\
& \left(C_{6}-C_{7}\right)\left(\mathcal{Q}^{2} \mathcal{Q}^{\dagger} \mathcal{Q}+\mathcal{Q}^{\dagger} \mathcal{Q} \mathcal{Q}^{\dagger 2}\right)
\end{aligned}
$$

However, one still needs to check whether this equation is compatible with (3).

Having listed the matrix representations of the parasupersymmetry generators for each energy level, i.e., Eqs. (35) and (36), the compatibility requirement may be enforced by substituting the matrix representations of $\mathcal{Q}, \mathcal{Q}^{\dagger}$ and $H$ in Eq. (38). This leads to a set of algebraic equations for the coefficients $C_{k}$.

Before pursuing the analysis of these equations, however, one must note that in view of the item 5 of the above list and the form of the Hamiltonian (37), the non-degenerate positive energy levels are not present in the spectrum. This is because such states,if existed, would have been annihilated by the right hand side of Eq. (38) and survived by the left hand side, leading to an obvious contradiction.

Next, consider the triply degenerate energy levels. In view of the analysis of the previous section, it is favorable to switch to a basis in which the chirality operator $\tau$ is diagonal. It is clear from (36) that $\tau$ has two eigenvalues $( \pm 1)$, one of them being degenerate. This 
allows one to have infinitely many choices for a unitary basis which diagonalizes $\tau$. In the following this arbitrariness is exploited to choose a basis in which the expressions for all the operators are considerably simplified. In fact, as it is demonstrated instantly, the value $\zeta=0$ is forbidden by the relation (37). Thus $\tau$ depends only on the conventional sign $\eta$ which can be set to + without loss of generality. Here it is assumed that the choice of $\eta$ is independent of the energy eigenvalue $E$.

In the new basis:

$$
e_{1}:=\left(\begin{array}{c}
\frac{-i t}{\sqrt{2}} \\
\sqrt{1-t^{2}} \\
\frac{i t}{\sqrt{2}}
\end{array}\right), \quad e_{2}:=\left(\begin{array}{c}
\sqrt{\frac{1-t^{2}}{2}} \\
-i t \\
-\sqrt{\frac{1-t^{2}}{2}}
\end{array}\right), \quad e_{2}:=\left(\begin{array}{c}
\frac{1}{\sqrt{2}} \\
0 \\
\frac{1}{\sqrt{2}}
\end{array}\right),
$$

where $t$ is defined by:

$$
t:=\epsilon \sqrt{1-\zeta^{2}} \in[-1,1]
$$

one has the following matrix representations:

$$
\begin{aligned}
\left.\tau\right|_{\mathcal{H}_{E}} & =\left(\begin{array}{ccc}
1 & 0 & 0 \\
0 & 1 & 0 \\
0 & 0 & -1
\end{array}\right), \\
\left.Q_{1}\right|_{\mathcal{H}_{E}} & =\sqrt{2 E}\left(\begin{array}{ccc}
0 & 0 & i t \\
0 & 0 & \sqrt{1-t^{2}} \\
-i t & \sqrt{1-t^{2}} & 0
\end{array}\right), \\
\left.Q_{2}\right|_{\mathcal{H}_{E}} & =\sqrt{2 E}\left(\begin{array}{ccc}
0 & 0 & 1 \\
0 & 0 & 0 \\
1 & 0 & 0
\end{array}\right) .
\end{aligned}
$$

Using these relations and Eq. (9), one then obtains the expressions for $\mathcal{Q}$ and $\mathcal{Q}^{\dagger}$. The latter may be substituted in the right hand side of Eq. (38). Equating the result with the left hand side which is just $E^{2}$ times the identity matrix, leads to four independent equations for the eight unknowns: $C_{k}, k=1, \cdots, 7$ and $t$ :

$$
\begin{aligned}
& \left(1-t^{2}\right)(1+t) C_{1}+\left(1-t^{2}\right)(1-t) C_{2}+(1+t)^{3} C_{3}+(1-t)^{3} C_{4}- \\
& 2\left(1-t^{2}\right)(1-t) C_{6}-2\left(1-t^{2}\right)(1+t)=\frac{1}{2}
\end{aligned}
$$




$$
\begin{aligned}
& \sqrt{1-t^{2}}\left[\left(1-t^{2}\right) C_{1}-\left(1-t^{2}\right) C_{2}+(1+t)^{2} C_{3}-(1-t)^{2} C_{4}+\right. \\
& \left.2 t(1-t) C_{6}+2 t(1+t) C_{7}\right]=0 \\
& \left(1-t^{2}\right)\left[(1-t) C_{1}+(1+t) C_{2}+(1+t) C_{3}+(1-t) C_{4}+\right. \\
& \left.2(1-t) C_{6}+2(1+t) C_{7}\right]=\frac{1}{2} \\
& (1-t)^{2} C_{3}+(1+t)^{2} C_{4}+2\left(1-t^{2}\right) C_{5}=\frac{1}{4} .
\end{aligned}
$$

One immediately concludes from Eq. (45) that the values $t= \pm 1$ (i.e., $\zeta=0, \epsilon= \pm 1$ ) are forbidden.

Eqs. (43)-(46) may be solved to express four of the unknowns in terms of the other four. For reasons which will be clear shortly, $t, x:=C_{3}, y:=C_{4}$ and $z:=C_{7}$ are chosen as independent variables. The solutions have a remarkably simple form:

$$
\begin{aligned}
& C_{1}=\frac{1-4(1+t)^{2} x}{4\left(1-t^{2}\right)}, \\
& C_{2}=\frac{1-4(1-t)^{2} y}{4\left(1-t^{2}\right)}, \\
& C_{5}=\frac{1-4(1-t)^{2} x-4(1+t)^{2} y}{8\left(1-t^{2}\right)}, \\
& C_{6}=\frac{-(1+t) z}{1-t} .
\end{aligned}
$$

At this stage, one must emphasize the role of the parameter $t$. As it is argued in [10], the defining parasuperalgebra does not impose any restrictions on the value of $t$. In fact, it may depend on the energy eigenvalue $E$. In general the value or values of $t$ may only be fixed if the detailed structure of the particular system of interest is known. For the systems admitting a Hamiltonian of the form (37), the coefficients $C_{k}$ are universal parameters independent of the energy eigenvalues. Existence of triply degenerate energy levels, however, make them dependent on $t$. Thus it is reasonable to assume that $t$ is also a universal (deformation) parameter taking a single value for all the triply degenerate energy levels. In fact, one can show the universality (uniqueness) of the parameter $t$ without making any additional assumption. To see this, let us assume that there is another parameter $t^{\prime} \in(-1,1)$ associated 
with some other energy eigenvalue $E^{\prime}>0$. Then the same analysis applies for $E^{\prime}$ and one obtains exactly the same equations as (47)-(50) with $t$ replaced by $t^{\prime}$. Introducing:

$$
\mathcal{C}:=\left(\begin{array}{c}
C_{1} \\
C_{2} \\
C_{5} \\
C_{6}
\end{array}\right), \quad \mathcal{X}:=\left(\begin{array}{c}
1 \\
x \\
y \\
z
\end{array}\right)
$$

one may rewrite Eqs. (47)-(50) as a matrix equation:

$$
\mathcal{C}=F(t) \mathcal{X}
$$

where $F(t)$ is a matrix whose value may be easily read from Eqs. (47)-(50). Since Eq. (51) must hold for both $t$ and $t^{\prime}$, one has:

$$
\left[F(t)-F\left(t^{\prime}\right)\right] \mathcal{X}=0
$$

However, by definition $\mathcal{X} \neq 0$. This implies the matrix $F(t)-F\left(t^{\prime}\right)$ to be singular, i.e.,

$$
\operatorname{det}\left[F(t)-F\left(t^{\prime}\right)\right]=0
$$

This equation can be easily solved. Its only solution is $t^{\prime}=t$. This concludes the proof that $t$ is independent of the energy eigenvalues.

In view of the uniqueness of $t$ and Eqs. (47)-(50), one may also assert that the moduli space $\mathcal{M}$ of the $(p=2)$ PSQM systems admitting a Hamiltonian of the form (37) and possessing triply degenerate energy levels, is $(-1,1) \times \mathbb{R}^{3} . \mathcal{M}$ has a subspace $\mathcal{N}$ corresponding to systems which include doubly degenerate energy levels as well. To construct $\mathcal{N}$, one may appeal to the matrix representations of the generators of the parasupersymmetry for the doubly degenerate levels, namely Eqs. (35). In view of these equations and relations (47)(50), one can easily show that the existence of doubly degenerate levels fixes $x:=C_{3}$ and $y:=C_{4}$ according to:

$$
x=y=\frac{1}{4} .
$$

This can be directly inferred from the original expression for the Hamiltonian (37). According to Eqs. (47)-(50) and (52), $\mathcal{N}$ may be identified with $(-1,1) \times \mathbb{R} \subset \mathcal{M}$. The subspace of $\mathcal{M}$ including the systems whose positive energy levels are all triply degenerate is then the 
set $\tilde{\mathcal{M}}:=\mathcal{M}-\mathcal{N}$. If one assumes that the chirality operator has the same representation for all the positive energy levels, i.e., $\tilde{\eta}=-\eta$ of (36) is independent of $E$, then the integer $\Delta^{(p=2)}$ of Eq. (6) is clearly a topological invariant for the elements of $\tilde{\mathcal{M}}$.

It must also be emphasized that by the topological invariance of $\Delta^{(p=2)}$ one means that if the Hamiltonian depends on a set of parameters $m \in M, \Delta^{(p=2)}$ is left unchanged under continuous variations of $m$. There is an obvious distinction between $\tilde{\mathcal{M}}$ and $M$. The latter may be an arbitrary (locally connected) topological (parameter) space that parameterizes the operator $\mathcal{Q}$ and therefore $H$. For example, one may take $M$ to be the space of all geometries on a given Riemannian manifold $X$, in which case $\Delta^{(p=2)}$ is a true topological invariant of $X$. On the other hand one may keep $\mathcal{Q}$ fixed and try to deform the Hamiltonian by continuously changing the parameters $t, x, y$ and $z$. In this case all values of the parameters belonging to $\tilde{\mathcal{M}}$ must yield the same value for $\Delta^{(p=2)}$, for $\tilde{\mathcal{M}}$ (with the subspace topology induced from the usual Euclidean metric topology on $\mathcal{M}$ ) is connected.

\section{Mathematical Interpretation of Parasupersymmet- ric Topological Invariants}

As it is argued in the preceding section, the topological content of the systems under investigation is independent of the free parameters $t, x, y$ and $z$ as far as they remain in $\tilde{\mathcal{M}}$. Therefore, in general one may fix one or some of these parameters in a topological investigation of PSQM. In the following, $t$ is chosen to vanish $(t=0)$ while the other parameters are kept free. In this case, the generators $Q_{1}$ and $Q_{2}$ have particularly simple expressions. According to (41) and (42), one has:

$$
\left.Q_{1}\right|_{\mathcal{H}_{E}}=\sqrt{2 E}\left(\begin{array}{ccc}
0 & 0 & 0 \\
0 & 0 & 1 \\
0 & 1 & 0
\end{array}\right),\left.\quad Q_{2}\right|_{\mathcal{H}_{E}}=\sqrt{2 E}\left(\begin{array}{ccc}
0 & 0 & 1 \\
0 & 0 & 0 \\
1 & 0 & 0
\end{array}\right) .
$$

In analogy with the case of SQM, as discussed in Sec. 2, Eqs. (53) may be employed to yield an algebraic expression for the topological invariant $\Delta^{(p=2)}$. In order to derive such an 
expression, first consider the following representation of the Hilbert space:

$$
\mathcal{H}=\left(\begin{array}{c}
\mathcal{H}_{+}^{1} \\
\mathcal{H}_{+}^{2} \\
\mathcal{H}_{-}
\end{array}\right), \text {with } \quad \mathcal{H}_{+}=:\left(\begin{array}{c}
\mathcal{H}_{+}^{1} \\
\mathcal{H}_{+}^{2}
\end{array}\right),
$$

where $\mathcal{H}_{+}$and $\mathcal{H}_{-}$are +1 and -1 eigenspaces of $\tau$ respectively.

In view of the constructions (20) and Eqs. (53), we propose:

$$
Q_{1}=\left(\begin{array}{ccc}
0 & 0 & 0 \\
0 & 0 & D_{1}^{\dagger} \\
0 & D_{1} & 0
\end{array}\right), \quad Q_{2}=\left(\begin{array}{ccc}
0 & 0 & D_{2}^{\dagger} \\
0 & 0 & 0 \\
D_{2} & 0 & 0
\end{array}\right),
$$

where $D_{i}: \mathcal{H}_{+}^{i} \rightarrow \mathcal{H}_{-}(i=1,2)$ are linear operators. Next we substitute the ansatz (55) in the parasuperalgebra (3) or alternatively (30)-(34) and Eq. (37) for the Hamiltonian.

Condition $\mathcal{Q}^{3}=0$ which in terms of $Q_{1}$ and $Q_{2}$ is expressed as Eqs. (30) and (31), together with the ansatz (55) lead to the following compatibility conditions:

$$
\left(D_{2} D_{2}^{\dagger}-D_{1} D_{1}^{\dagger}\right) D_{i}=0 \quad(i=1,2) .
$$

These conditions, in turn simplify the expression (38) for the Hamiltonian which then reads:

$$
H^{2}=\frac{1}{2}\left(\begin{array}{ccc}
\gamma_{1}\left(D_{2}^{\dagger} D_{2}\right)^{2} & i \gamma_{2} D_{2}^{\dagger} D_{1} D_{1}^{\dagger} D_{1} & 0 \\
-i \gamma_{2} D_{1}^{\dagger} D_{1} D_{1}^{\dagger} D_{2} & \gamma_{1}\left(D_{1}^{\dagger} D_{1}\right)^{2} & 0 \\
0 & 0 & \gamma_{3}\left(D_{1} D_{1}^{\dagger}+D_{2} D_{2}^{\dagger}\right)^{2}+\gamma_{4}\left[\left(D_{1} D_{1}^{\dagger}\right)^{2}-\left(D_{2} D_{2}^{\dagger}\right)^{2}\right]
\end{array}\right)
$$

where

$$
\begin{aligned}
\gamma_{1} & :=C_{1}+C_{2}+C_{3}+C_{4}-2 C_{6}-2 C_{7}, \\
\gamma_{2} & :=C_{1}-C_{2}+C_{3}-C_{4}, \\
\gamma_{3} & :=\frac{C_{3}}{2}+C_{4} 2+C_{5}, \quad \gamma_{4}:=C_{6}+C_{7} .
\end{aligned}
$$

Taking $t=0$ in Eqs. (47)-(50) and substituting the result in (58), one finds

$$
\gamma_{1}=\frac{1}{2}, \quad \gamma_{2}=\gamma_{4}=0, \quad \gamma_{3}=\frac{1}{8} .
$$


The fact that $\gamma$ 's are independent of the variables $x, y$, and $z$ is quite remarkable. In view of these results, one can easily take the square root of both sides of (57) to yield:

$$
H=\frac{1}{2}\left(\begin{array}{ccc}
D_{2}^{\dagger} D_{2} & 0 & 0 \\
0 & D_{1}^{\dagger} D_{1} & 0 \\
0 & 0 & \frac{1}{2}\left(D_{1} D_{1}^{\dagger}+D_{2} D_{2}^{\dagger}\right)
\end{array}\right)
$$

Another remarkable observation is that indeed the Hamiltonian as expressed by Eq. (59) also satisfies the other parasuperalgebra relations, namely Eq. (3) or alternatively Eqs. (32)-(34). This is also highly nontrivial.

Having obtained the expression for the Hamiltonian in a basis which explicitly distinguishes the odd and even chirality states, one can easily derive the formula for $\Delta^{(p=2)}$ :

$$
\Delta^{(p=2)}=\operatorname{dim}\left(\operatorname{ker} D_{1}\right)+\operatorname{dim}\left(\operatorname{ker} D_{2}\right)-2 \operatorname{dim}\left(\operatorname{ker} D_{1}^{\dagger} \cap \operatorname{ker} D_{2}^{\dagger}\right) \text {. }
$$

Here we have employed the following identifications:

$$
\begin{aligned}
n_{0}^{\pi B} & =\operatorname{dim}\left(\operatorname{ker} D_{1}^{\dagger} D_{1} \oplus \operatorname{ker} D_{2}^{\dagger} D_{2}\right) \\
& =\operatorname{dim}\left(\operatorname{ker} D_{1}\right)+\operatorname{dim}\left(\operatorname{ker} D_{2}\right) \\
n_{0}^{\pi F} & =\operatorname{dim}\left(\operatorname{ker}\left[D_{1} D_{1}^{\dagger}+D_{2} D_{2}^{\dagger}\right]\right) \\
& =\operatorname{dim}\left(\operatorname{ker} D_{1}^{\dagger} \cap \operatorname{ker} D_{2}^{\dagger}\right) .
\end{aligned}
$$

In Eqs. (61) and (62) use is made of relations (26).

It turns out that conditions (56) may be used to simplify the expression (60) for $\Delta^{(p=2)}$. To see this let us define $A_{i}:=D_{i} D_{i}^{\dagger}$, $(\mathrm{i}=1,2)$. Then multiplying Eqs. (56) by $D_{i}^{\dagger}$ from the right and writing the resulting equations in terms of $A_{i}$, one has:

$$
\left(A_{1}-A_{2}\right) A_{1}=0, \quad\left(A_{1}-A_{2}\right) A_{2}=0 .
$$

In view of the fact that $A_{i}$ are self-adjoint and positive (semi)definite operators, Eqs. (63) imply $\operatorname{ker} A_{1}=\operatorname{ker} A_{2}$. This together with the identities (26) lead to $\operatorname{ker} D_{1}^{\dagger}=\operatorname{ker} D_{2}^{\dagger}$. Thus, we have:

$$
\Delta^{(p=2)}=\operatorname{index}^{\text {analytic }}\left(D_{1}\right)+\operatorname{index} \operatorname{analytic}^{(}\left(D_{2}\right) .
$$

Eq. (64) provides the desired mathematical interpretation for the parasupersymmetric topological invariant (6). 
It must be emphasized that Eq. (64) is only valid for the ansatz (55). In fact the most general expression which relates $Q_{1}$ and $Q_{2}$ with a pair of linear operators $D_{1}$ and $D_{2}$ is (20), where the representation (19) is used for the Hilbert space. In this case however, enforcing the R-S PSQM algebra, one is led to complicated compatibility conditions between $D_{1}$ and $D_{2}$ which render a similar approach ineffective.

\section{Conclusion}

The $(p=2)$ parasupersymmetric quantum mechanics may be viewed as a generalization of the ordinary supersymmetric quantum mechanics. A study of the spectrum degeneracy structure of the $(p=2)$ parasupersymmetry leads to the definition of a topological invariant. For a class of $(p=2)$ parasupersymmetric systems this invariant may be given a well-known mathematical meaning, namely that it is associated with the sum of analytical indices of a pair of Fredholm operators. In a sense, this is a negative result as one might have hoped for a more general and possibly new topological invariant.

Unlike supersymmetric quantum mechanics, the form of the Hamiltonian is not determined by the defining algebraic relations in parasupersymmetric quantum mechanics. Thus in general one needs to investigate possible forms of the Hamiltonian which are compatible with the defining parasuperalgebras of $(p=2)-$ PSQM and attempt to classify the corresponding systems. In the present article it is shown how the matrix representations of the relevant operators, which are valid for any quantum system satisfying the definition of PSQM, may be used to classify the forms of the corresponding Hamiltonians. This is carried out explicitly for the case where the Hamiltonian is the square root of a forth order polynomial in the generators. However the method enjoys general applicability.

For the particular class (37) of the Hamiltonians considered here, it is shown that the parameter $t=\epsilon \sqrt{1-\zeta^{2}}$ of the R-S PSQM is a universal parameter, i.e., it is independent of the energy eigenvalues.

The mathematical interpretation of parasupersymmetric topological invariant $\Delta^{(p=2)}$ offered in this article depends on the ansatz (55) chosen to relate the generators of parasupersymmetry with some Fredholm operators. This is justified by making analogy with the case of supersymmetry and assuming the parameter $t$ to vanish. In fact, if one does retain the ansatz (55) but considers the case $t \neq 0$, then the parasuperalgebra relations are not as 
trivially satisfied. In fact, in this case, Eq. (57) remains valid but the compatibility with Eq. (3) leads to:

$$
\gamma_{1}-\gamma_{2}=\frac{1}{2}, \quad \gamma_{3}=\frac{1}{8}
$$

These equations are obtained by multiplying both sides of Eq. (3) by $H$ and using the same equation to express the left hand side of the result in terms of $\mathcal{Q}$ and $\mathcal{Q}^{\dagger}$. This yields an equation involving $H^{2}, \mathcal{Q}$ and $\mathcal{Q}^{\dagger}$ which upon substitution of (9), (55) and (57) results in (65). In view of the definition of $\gamma$ 's (58), the latter equations add to Eqs. (43)-(46). This indicates that the ansatz (55) is valid for a proper subset of $\tilde{\mathcal{M}}$ consisting of the sector corresponding to $t=0$ and one defined by the simultaneous solution of Eqs. (43)-(46) and (65) with $t \neq 0$.

The methods and ideas developed in this article may be easily applied for the B-D PSQM. In fact, requiring the Hamiltonian to have the form (37) and using the matrix representations of the generators of the B-D PSQM [10], one recovers the same equations for the coefficients, i.e., (47)-(50), with $t=0$. Therefore the results obtained in sections 3 and 4 for the R-S PSQM with $t=0$ are also valid for the B-D PSQM systems in general.

\section{Acknowledgements}

Parts of this research were carried out at the Institute for Studies in Theoretical Physics and Mathematics (IPM) at Tehran. The author would like to thank V. Karimipour, S. Rouhani of the IPM, and A. Rezaii of Tabriz University for invaluable comments and discussions. He also appreciates the financial support of the Killam foundation of Canada. 


\section{References}

[1] M. F. Atiyah and I. M. Singer, Bull. Amer. Math. Soc. 69, 422 (1963); Ann. Math. 87,546 (1968); See also [2, 3].

[2] R. S. Palais, "Seminar on the Atiyah-Singer Index Theorem," Ann. of Math. Study, Vol. 57, Princeton Uni. Press, Princeton (1965).

[3] P. Shanahan, "The Atiyah-Singer Index Theorem," Lect. Notes in Math. Vol. 638, Springer, New York (1978).

[4] E. Witten, Nucl. Phys. B202, 253 (1982).

[5] L. Alvarez-Gaume, Commun. Math. Phys. 90, 161 (1983); J. Phys. A: Math. Gen. 16, 4177 (1983); P. Windey, Acta Physica Polonica B15, 453 (1984); For a more complete list of references see [6].

[6] A. Mostafazadeh, J. Math. Phys. 35, 1095 (1994).

[7] V. A. Rubakov and V. P. Spiridonov, Mod. Phys. Lett. A3, 1337 (1988).

[8] A. Khare, J. Phys. A: Math. Gen. 25, L749 (1992).

[9] J. Beckers and N. Debergh, Nucl. Phys. B340, 767 (1990).

[10] A. Mostafazadeh, Int. J. Mod. Phys. A11, 1057 (1996).

[11] A. Khare, A. K. Mishra, and G. Rajasekaran, Mod. Phys. Lett. A8, 107 (1993). 